\title{
Ceratoplastia autóloga ipsilateral rotacional em ceratocone após 24 meses de seguimento
}

\author{
Refractive and topographic changes of ipsilateral rotational autokeratoplasty with a crescent-shape \\ $0.5 \mathrm{~mm}$ resection of the inferior cornea in keratoconus after a 24 months follow-up
}

Fernando J. M. Cunha ${ }^{1}$, Wagner Lira ${ }^{2}$, Carlos Teixeira Brandt ${ }^{3}$

\section{RESUMO}

Introdução: A ceratoplastia penetrante autóloga ipsilateral rotacional pode ser uma alternativa para minimizar o problema de longas filas de espera para transplante por ceratocone

Objetivo: Apresentar os resultados visuais em portadores de ceratocone após ceratoplastia penetrante autóloga ipsilateral rotacional associada à ressecção de 0,5 mm de crescente corneana inferior.

Métodos: Estudo prospectivo, longitudinal, de intervenção, analítico, investigando os resultados visuais de 15 pacientes (18 olhos), com diagnóstico de ceratocone, submetidos a ceratoplastia penetrante autóloga ipsilateral rotacional (CPAIR) por meio de duas trepanações, coincidentes superiormente e com diferença de 0,5 mm inferiormente. Após ressecção da cunha resultante das duas trepanações inferiores, o disco corneano foi rotado de $180^{\circ}$ e suturado com 24 pontos diametralmente opostos. Os pacientes foram acompanhados por um período de 24 meses.

Resultados: Ao comparar os dados de $1^{\circ}$ e $2^{\circ}$ anos de pós-operatório aos do préoperatório, observou-se melhora significante de: acuidade visual, linhas de visão, equivalente esférico, diminuição do astigmatismo topográfico e das curvaturas corneais máxima e média. Houve adelgaçamento corneano, com diminuição do número de células endoteliais da córnea.

Conclusão: Observou-se melhora dos parâmetros oftalmológicos decorridos 6, 12 e 24 meses da ceratoplastia penetrante autóloga ipsilateral rotacional associada à ressecção de 0,5 $\mathrm{mm}$ na porção inferior corneana, mas ao longo do seguimento houve redução da acuidade visual com aumento do astigmatismo.

Descritores: Ceratocone/diagnóstico; Ceratoplastia penetrante/métodos; Córnea/ fisiopatologia; Visão binocular/fisiologia. Transplante autólogo; Acuidade visual; Seguimentos

\section{ABSTRACT}

Introduction: Ipsilateral rotational penetrating autokeratoplasty technique may be an alternative to minimize the problem of long waiting lists for transplantation due to keratoconus.

Purpose: To report the visual outcomes in keratoconus patients afteripsilateral rotational penetrating autokeratoplastytechnique (IRPAK) associated with acrescent-shaperesection of $0.5 \mathrm{~mm}$ of the inferior cornea.

Methods: According to a prospective, longitudinal, interventional, analytic study, the authors investigated the visual results of 15 patients (18 eyes) with keratoconus, who underwent an ipsilateral rotational penetrating autokeratoplasty, by two coincident trepanations superiorly and with a difference of $0.5 \mathrm{~mm}$ inferiorly. After resection of this corneal crescent, a $180^{\circ}$ rotation of the corneal disk was performed, which was fixed with 24 sutures diametrically opposed. The patients were followed-up for a period of 24 months.

Results: Comparing data of the first and second postoperative years to preoperative data, there were: significant improvements in visual acuity, vision lines and spherical equivalent; reduction of topographic astigmatism and maximum and mean corneal curvatures. There was also a decrease in corneal thickness associated to a reduction of corneal endothelial cell count.

Conclusion: There was an improvement of ophthalmologic parameters 6, 12 and 24 months after ipsilateral rotational penetrating autokeratoplasty associated with a crescent-shape resection of $0.5 \mathrm{~mm}$ of the inferior cornea, but along the follow-up there was reduction of visual acuity with increased astigmatism.

Keywords: Keratoconus/diagnosis; Keratoplasty, penetrating/methods; Cornea/physiopathology; Vision, binocular/physiology; Transplantation, autologous; Visual acuity; Follow-up studies

\section{INTRODUÇÃO}

O ceratocone tem sido a indicação mais frequente para ceratoplastia penetrante em centros de referência no mundo e no Brasil, incluindo a Fundação Altino Ventura na cidade de Recife - Pernambuco(1).

A doença inicia-se, geralmente, na segunda ou terceira década de vida, incapacitando indivíduos, devido à deficiência visual secundária à deformidade corneana (2). Pode evoluir para um astigmatismo irregular, passível de correção e permitindo boa acuidade visual com uso de óculos ou lente de contato rígida, ou evoluir para uma situação de adelgaçamento e protrusão avançados, inviabilizando a correção pelos recursos ópticos existentes. Nesses casos, está indicada a realização de transplante de córnea, o que ocorre em $10 \%$ a $20 \%$ dos pacientes ${ }^{(3,4)}$

A espera por um transplante em países em desenvolvimento é prolongada(4). Apesar de em algumas regiões brasileiras esse problema estar solucionado, segundo os dados do Banco de Olhos do Recife, em outubro de 2010, havia 1.139 inscritos na lista de espera para transplante de córnea, a serem atendidos num tempo médio de quatro a cinco anos ${ }^{(5)}$.

O transplante rotacional autólogo tem sido descrito como boa alternativa no manejo de opacidades corneanas centrais, principalmente em países onde não se encontra disponibilidade de tecido corneano para doação. Apresenta ainda, a vantagem de não induzir
Submitted for publication: December 22,2010

Accepted for publication: June 11, 2011

Study carried out at the Fundação Altino Ventura (FAV) - Recife - PE.

Physician, Departamento de Córnea e Doenças Externas do Hospital da Visão, Recife (PE), Brazil.

2 Physician, Universidade Federal de Pernambuco - UFPE- Recife (PE), Brazil.

${ }^{3}$ Physician, Departamento de Córnea e Doenças Externas, Fundação Altino Ventura, Recife (PE),

Brazil.
Funding: No specific financial support was available for this study.

Disclosure of potential conflicts of interest: Cunha FJM, None; Lira W, None; Brandt CT, None.

Correspondence address: Fernando J. M. Cunha. Av. Boa Viagem, 2.080 - Apto. 1.901 - Recife PE - 51011-000 - Brazil - E-mail: fernandojmcunha@uol.com.br 
rejeição, que é uma das principais causas de insucesso nos transplantes de córnea ${ }^{(6,7)}$.

O uso da autoceratoplastia ipsilateral rotacional para opacidades corneanas tem produzido resultados bons e regulares, com média de tempos de seguimento de cerca de 12 meses $^{(4,6,6,8)}$.

O objetivo do estudo foi apresentar os resultados de acuidade visual, dados refracionais e topográficos de portadores de ceratocone submetidos a ceratoplastia penetrante autóloga ipsilateral rotacional associada à ressecção de $0,5 \mathrm{~mm}$ de crescente corneana inferior, após seguimento de dois anos.

\section{MÉTODOS}

Foi realizado estudo prospectivo, longitudinal, de intervenção, analítico, envolvendo 15 pacientes (18 olhos) com ceratocone, submetidos a ceratoplastia penetrante autóloga ipsilateral rotacional (CPAIR) na Fundação Altino Ventura, no período de agosto de 2004 a junho de 2009.

Foram incluídos os pacientes com ceratocone, inscritos no Banco de Olhos, que apresentavam contraindicação para o uso de lentes de contato, por alergia ocular, má adaptação ou condições sociais desfavoráveis. Esses pacientes apresentavam acuidade visual corrigida por uso de óculos pior ou igual que 20/200 (logMAR 1,0) ou corrigida por lente de contato rígida pior ou igual a 20/80 (logMAR 0,602).

Foram excluídos os pacientes com ceratocone do tipo globoso ou com cicatrizes estromais secundárias a hidropsia corneana, assim como os portadores de glaucoma ou doenças do segmento posterior. Foram também excluídos pacientes com doença de base que pudesse comprometer o processo cicatricial e aqueles com história de cirurgias oculares prévias.

Dez $(66,7 \%)$ pacientes do sexo feminino e 5 (33,3\%) do masculino, eram procedentes do estado de Pernambuco, inscritos no Banco de Olhos de Pernambuco entre março de 2002 e março de 2006. A média de idades foi de $25,7 \pm 2,3$ anos, variando entre 13,9 anos e 50,9 anos, com mediana de 24 anos.

Os pacientes foram submetidos à ceratoplastia penetrante autóloga ipsilateral rotacional, sendo nove olhos direitos e nove esquerdos. Doze (80\%) pacientes tiveram um olho operado e 3 (20\%) pacientes tiveram ambos os olhos submetidos ao procedimento cirúrgico.

Os pacientes foram submetidos a exame oftalmológico composto por: aferição da acuidade visual com e sem correção, utilizando tabela de Snellen a seis metros de distância, refratometria, biomicroscopia, tonometria de aplanação, fundoscopia e ceratoscopia computadorizada, em topógrafo Eye Sys (versão 3.10).

Nos pacientes com indicação de CPAIR, procedeu-se ao planejamento cirúrgico em lâmpada de fenda para determinar o diâmetro dos trépanos e localização da trepanação, guiada também pela topografia. Realizou-se trepanação centrada para o ceratocone mamilar e trepanação abrangendo a periferia superior, para ceratocone descentrado temporal inferior. Utilizaram-se dois trépanos de diâmetros diferentes: um de diâmetro capaz de abranger todo o ceratocone e a periferia corneana superior, e outro, capaz de poupar $0,5 \mathrm{~mm}$ do limbo inferior.

Após anestesia peribulbar, foram realizadas duas trepanações coincidentes superiormente e com diferença de 0,5 $\mathrm{mm}$ inferiormente, o que permitiu a ressecção de um segmento corneano, em forma de crescente, na região inferior mais próxima ao afinamento, com a finalidade de promover maior aplanação do disco corneano. Foi realizada rotação do disco corneano a $180^{\circ}$ deslocando o ápice do cone para a região superior e a região mais espessa da córnea, para o eixo visual. O botão foi suturado utilizando fio mononylon 10.0 com 24 pontos diametralmente separados. Ao final da cirurgia, foi realizada ceratoscopia com disco de Plácido e substituição dos pontos frouxos ou apertados (Figura 1).

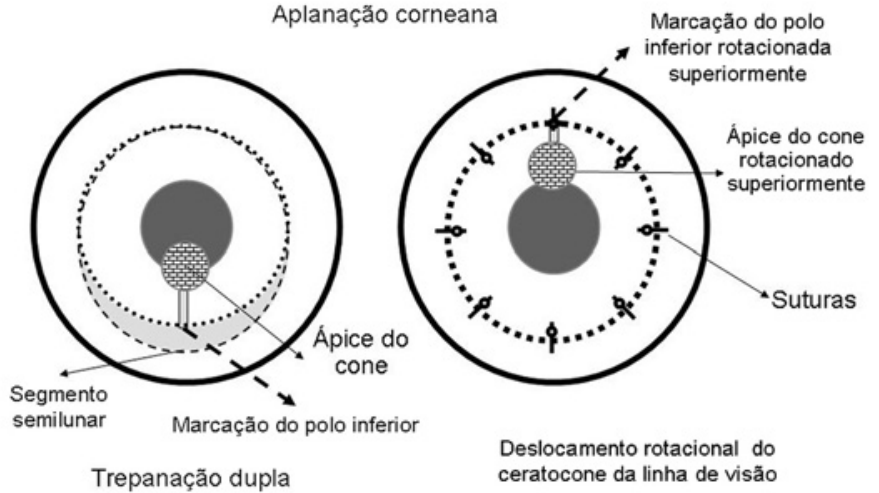

Figura 1. Esquema da aplanação corneana empregada na CPAIR. À esquerda, demonstrativo da dupla trepanação corneana, com segmento semilunar de 0,5 mm. À direita, demonstração da rotação do botão corneano, posicionando o ápice do ceratocone superiormente.

Foram prescritos, no pós-operatório, colírios de ofloxacino a 0,3\% por um período de 21 dias e acetato de prednisolona a 1\%, por seis semanas. Em ambulatório, no acompanhamento pós-operatório, procedeu-se ao exame oftalmológico mensal composto por aferição da acuidade visual, utilizando tabela de Snellen, biomicroscopia e tonometria de aplanação.

A partir do terceiro mês pós-operatório, foram realizadas refração e topografia corneal de todos os pacientes, a cada três meses, cujos resultados serviram de orientação para a remoção seletiva de suturas indutoras de astigmatismo. Após a retirada das suturas, os pacientes foram acompanhados por 24 meses.

Os resultados foram expressos por médias. Para avaliação dos resultados obtidos com a CPAIR, foram comparados os parâmetros oftalmológicos iniciais com aqueles decorridos seis, 12 e 24 meses. Foi usado o teste de Wilcoxon pareado de diferenças em amostras relacionadas. O nível de significância foi estabelecido em $p<0,050$.

O trabalho foi aprovado pelo Comitê de Ética da Fundação Altino Ventura e os pacientes assinaram o Termo de Consentimento Livre Esclarecido.

\section{RESULTADOS}

Na tabela 1, estão expressas as avaliações médias iniciais e decorridos seis, 12 e 24 meses da CPAIR, constatando-se melhora da acuidade visual (expressa em logMAR), ganho de linhas de visão, melhora do equivalente esférico, diminuição do astigmatismo topográfico e das curvaturas corneanas máxima e média, assim como adelgaçamento corneano. Também se verificou diminuição do número de células endoteliais corneanas, decorridos seis, 12 e 24 meses da CPAIR, quando comparado ao inicial.

\section{COMPARAÇÃo dOS PARÂMETROS OFTALMOLÓGICOS DECORRIDOS 24 MESES DA CPAIR}

Considerando a acuidade visual inicial de cada um dos 18 olhos como seu padrão, houve melhora significante em cada uma das avaliações, em relação à $A V L$ inicial, conforme demonstrado nas tabelas 2 a 4. Constatou-se que nenhum paciente apresentou piora da acuidade visual, decorridos seis meses da CPAIR e o ganho de linhas de visão variou de quatro (passando a AVL de conta-dedos a 1,0 m para 20/200) a 16 (com variação da AVL de conta-dedos a 2 m para 20/25). Após 12 meses, 17 (94,4\%) pacientes tiveram ganho de linhas de visão, variando de duas linhas (com AVL passando de 20/200 para 20/100) a 16 linhas (em um paciente que manteve AVL igual a 20/25). Nessa avaliação, um paciente apresentou piora da acuidade visual (20/400) em relação à inicial (20/200). Aos 24 meses, mais dois pacientes apresentavam acuidade visual igual à inicial (mantendo 20/400) (Tabela 2). 
Tabela 1. Distribuição das avaliações de 18 olhos submetidos a CPAIR, segundo época das aferições - Recife - agosto 2004 - junho 2009

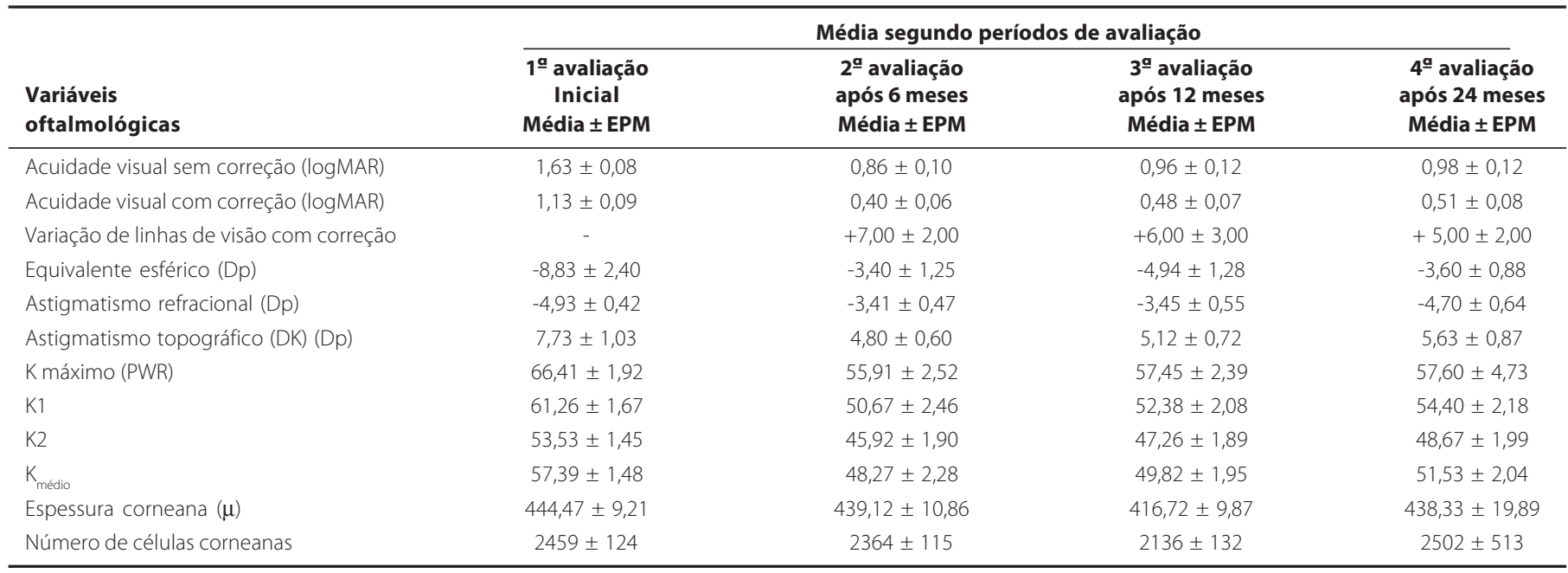

Nota: Um paciente não pode ter o equivalente esférico aferido ao primeiro exame devido à irregularidade corneana. Essa irregularidade acentuou-se para dois pacientes na $3^{\mathfrak{a}}$ avaliação e em três casos, na $4^{\underline{\underline{a}}}$ avaliação. Não puderam ser aferidos após a CPAIR=DK de dois pacientes na $2^{\underline{\underline{a}}}$ avaliação e um paciente na $4^{\underline{\underline{a}}}$ avaliação: $\mathrm{K}$ $\mathrm{PIO}$ de três pacientes, na $2^{\underline{\underline{a}}}$ avaliação e de um paciente na $3^{\underline{a}}$ e $4^{\underline{a}}$ avaliações; $\mathrm{K} 1$ de dois pacientes, na $2^{\underline{a}}$ avaliação e um paciente na $3^{\underline{\underline{a}}}$ e $4^{\underline{\underline{a}}}$ avaliações, $\mathrm{K} 2$, de um paciente na $4^{\underline{\underline{a}}}$ avaliação; espessura corneana de um paciente, na $2^{\underline{a}}$ e $3^{\underline{a}}$ avaliações e de três pacientes na $4^{\underline{a}}$; número de células de seis pacientes na $2^{\underline{a}}$ avaliação, quatro casos, na $3^{\underline{a}}$. e cinco casos na $4^{\underline{a}}$, devido à irregularidade corneana

Ao comparar cada avaliação com a subsequente, observou-se que na avaliação após 12 meses da cirurgia, 8 (44,4\%) olhos tiveram perda de linhas de visão, variando de uma a oito linhas. Um paciente alcançara AVL de 20/50, na avaliação após seis meses, a qual se reduziu a 20/400, após 12 meses da cirurgia, o que o caracterizou como o único resultado do estudo com AVL final menor que o inicial.

Quanto ao astigmatismo topográfico, decorridos seis meses da CPAIR, em 14 dos 16 olhos aferidos (87,5\%) houve redução do parâmetro, variando de $-0,01$ a $-13,0$ Dp, em relação à avaliação préoperatória, enquanto que 2 (12,5\%) apresentaram aumento desse parâmetro. As reduções medianas alcançaram significância estatística, decorridos seis e 12 meses da CPAIR, em relação ao valor inicial. No entanto, ao longo do tempo, um número crescente de olhos teve aumento do astigmatismo, de tal forma que, após 24 meses da cirurgia, 11 dos 17 (64,7\%) olhos aferidos apresentavam maior astigmatismo que o valor determinado após 12 meses da cirurgia (Tabela 2). Mesmo assim, em nenhum dos olhos a aferição após 24 meses atingiu o valor pré-operatório.

Houve melhora significante do equivalente esférico entre a primeira avaliação e as demais, com variação predominante entre -10,55 Dp e -0,50 Dp. Também foi significante o aumento do equivalente esférico entre a aferição após seis meses da CPAIR e a realizada após 12 meses da cirurgia, porém a valores maiores que o inicial (Tabela 1). A redução do equivalente esférico ocorreu em 16 $(94,1 \%)$ dos 17 olhos aferidos após seis meses da cirurgia, em 15 (93,8\%) dos 16 olhos após 12 meses da CPAIR e em 100\% dos 15 olhos, na última avaliação. Ao comparar cada aferição à subsequente, constatou-se aumento do equivalente esférico em $9(69,2 \%)$ de 13 olhos entre seis e 12 meses após a cirurgia, porcentual que se reduziu a 26,7\% entre esta avaliação e a última, após 24 meses (Tabela 2).

Comparando-se as aferições do valor ceratométrico máximo simulado ( $K_{\text {máximo }}$ ) após seis meses da cirurgia, com o valor inicial, constatou-se que 16 dos 17 olhos aferidos (94,1\%) apresentavam redução do $K_{\text {máximo' }}$ porcentual que diminuiu apenas no segundo ano da CPAIR, para 14 dos 16 olhos (87,5\%). No entanto, ao comparar cada aferição à subsequente, identificou-se que o ganho inicial foi se reduzindo, de tal forma que aos 12 meses, 10 dos 16 olhos
(62,5\%) apresentavam aumento do valor ceratométrico máximo simulado de até $10 \mathrm{Dp}$, acentuando essa diferença, decorridos 24 meses (Tabela 2).

Em relação aos parâmetros ceratométricos, conforme apresentado na Tabela 3, mais frequentemente se observou redução em porcentuais próximos ou iguais a 100\% dos olhos, nas três avaliações, quando comparadas à aferição pré-operatória, em valores variando de $-30,00$ a $-0,01$. Todas essas diferenças alcançaram significância estatística. Apesar disso, quando se determinaram as variações de uma avaliação para outra, constatou-se aumento gradual desses valores. Da comparação entre a aferição após 12 meses com aquela realizada após seis meses da CPAIR, observou-se que em 11 dos 16 olhos aferidos (68,8\%) houve aumento de K1, o qual se manteve na comparação feita entre esta e a avaliação subsequente. Comportamento semelhante foi verificado para a aferição de K2. Foram significantes as variações de $\mathrm{K}_{1}$ e $\mathrm{K}_{\text {médi }}$ entre seis meses e 12 meses, enquanto que a variação de K2 foi significante no período entre 12 e 24 meses (Tabela 3).

Foram significantes as reduções da espessura corneana ocorridas após 12 meses da cirurgia, quando comparadas aos valores iniciais e depois de seis meses da CPAIR, assim como foi significante o aumento da espessura que se verificou após 24 meses, quando comparada àquela dos 12 meses, como demonstrado nas tabelas 1 e 4. Decorridos seis meses da cirurgia, oito dos 17 olhos (47\%) tinham aumento da espessura corneana, mas, após 12 meses, esse porcentual reduziu-se a 17,6\%, voltando a alcançar o mesmo porcentual de 47\%, na comparação entre a terceira e a quarta avaliações.

A esse fato, associou-se redução significante do número de células endoteliais corneanas decorridos seis meses, verificada em oito dos 12 olhos aferidos (75\%), como também após 12 meses de cirurgia, em 10 dos 14 olhos aferidos (71,4\%) e 12 dos 13 olhos aferidos (92,3\%), na avaliação após 24 meses da cirurgia. Essas variações embora tenham ocorrido com frequência, não alcançaram significância estatística. É importante ressaltar que em seis dos 12 olhos aferidos, na comparação entre a avaliação de seis meses e a subsequente, foi diagnosticado aumento do número de células (Tabela 4).

No quadro 1, estão resumidas as alterações significantes, apresentadas com o intuito de facilitar a análise dos resultados.

Foram observadas complicações em três pacientes. Um caso apresentou ruptura de dois pontos por trauma ocular e Seidel, que 
Tabela 2. Distribuição das variações dos parâmetros de 18 olhos submetidos a CPAIR, segundo época das aferições - Recife agosto 2004 - junho 2009

\begin{tabular}{|c|c|c|c|c|c|c|}
\hline \multicolumn{2}{|c|}{$\begin{array}{l}\text { Variações dos parâmetros } \\
\text { nas avaliações }\end{array}$} & $\begin{array}{l}\text { de } 6 \text { meses - } \\
\text { inicial }\end{array}$ & $\begin{array}{l}\text { de } 12 \text { meses - } \\
\text { inicial }\end{array}$ & $\begin{array}{l}\text { de } 24 \text { meses - } \\
\text { inicial }\end{array}$ & $\begin{array}{l}\text { de } 12 \text { meses - } \\
6 \text { meses }\end{array}$ & $\begin{array}{l}\text { de } 24 \text { meses - } \\
12 \text { meses }\end{array}$ \\
\hline \multicolumn{7}{|c|}{ Variação da AVL em linhas de visão } \\
\hline \multirow{2}{*}{ Redução } & $-8 a-3$ & 0 & 0 & 0 & 4 & 3 \\
\hline & $-2 a-1$ & 0 & 1 & 1 & 4 & 3 \\
\hline Manutenção & 0 & 0 & 0 & 0 & 6 & 6 \\
\hline \multirow[t]{3}{*}{ Ganho } & $+1 a+3$ & 0 & 3 & 4 & 3 & 5 \\
\hline & $+4 a+7$ & 12 & 8 & 8 & 1 & 1 \\
\hline & $+8 a+16$ & 6 & 6 & 5 & 0 & 0 \\
\hline \multicolumn{7}{|c|}{ Variação do astigmatismo refracional (Dp) } \\
\hline \multirow[t]{2}{*}{ Aumento } & $-6,00$ a $-4,00$ & 0 & 0 & 0 & 0 & 3 \\
\hline & $-3,00$ a $-0,25$ & 2 & 5 & 4 & 8 & 4 \\
\hline Manutenção & 0 & 5 & 5 & 9 & 7 & 6 \\
\hline \multirow[t]{3}{*}{ Redução } & $+0,50 \mathrm{a}+1,75$ & 6 & 2 & 2 & 5 & 3 \\
\hline & $+2,00 \mathrm{a}+4,75$ & 3 & 4 & 3 & 0 & 2 \\
\hline & $+5,00 \mathrm{a}+6,00$ & 2 & 2 & 0 & 0 & 0 \\
\hline \multicolumn{7}{|c|}{ Variação do astigmatismo topográfico (Dp)' } \\
\hline \multirow[t]{3}{*}{ Redução } & $-13,00$ a $-6,00$ & 4 & 6 & 4 & 0 & 0 \\
\hline & $-5,99$ a $-2,00$ & 4 & 5 & 5 & 3 & 3 \\
\hline & $-1,99$ a $-0,01$ & 6 & 2 & 3 & 5 & 3 \\
\hline \multirow[t]{2}{*}{ Aumento } & $+0,01 \mathrm{a}+1,99$ & 2 & 2 & 2 & 6 & 7 \\
\hline & $+2,00 \mathrm{a}+7,00$ & 0 & 3 & 3 & 2 & 4 \\
\hline \multicolumn{7}{|c|}{ Variação do equivalente esférico (Dp) } \\
\hline \multirow[t]{2}{*}{ Redução } & $-17,00$ a $-8,00$ & 8 & 6 & 8 & 0 & 0 \\
\hline & $-7,99$ a $-0,01$ & 8 & 9 & 6 & 3 & 11 \\
\hline Manutenção & 0 & 0 & 0 & 0 & 1 & 0 \\
\hline \multirow[t]{2}{*}{ Aumento } & $+0,01 \mathrm{a}+7,99$ & 1 & 1 & 0 & 8 & 4 \\
\hline & $+8,00 \mathrm{a}+17,00$ & 0 & 0 & 0 & 1 & 0 \\
\hline \multicolumn{7}{|c|}{ Variação do valor ceratométrico máximo (PWR) (Dp) } \\
\hline \multirow[t]{2}{*}{ Redução } & $-60,00$ a $-10,00$ & 7 & 8 & 3 & 0 & 1 \\
\hline & $-9,99$ a $-0,01$ & 9 & 8 & 11 & 6 & 4 \\
\hline \multirow[t]{2}{*}{ Aumento } & $+0,01 \mathrm{a}+10,00$ & 1 & 1 & 1 & 10 & 9 \\
\hline & $+10,01$ a $+20,00$ & 0 & 0 & 1 & 0 & 2 \\
\hline
\end{tabular}

Nota: Um paciente não pode ter o equivalente esférico aferido ao primeiro exame devido à irregularidade corneana. Essa irregularidade acentuou-se para dois pacientes na $3^{\underline{a}}$ avaliação

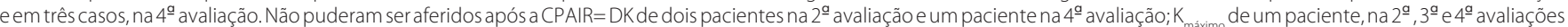

foram repostos. Em outro, foi diagnosticado Seidel passivo inferior, no sétimo dia de pós-operatório, tendo sido submetido a nova sutura. O terceiro paciente apresentou sinéquia anterior localizada às 12 horas.

\section{DISCUSSÃO}

A técnica de autoceratoplastia ipsilateral tem sido esporadicamente referida na literatura, embora tenha sido descrita desde 1967 para correção de cicatrizes corneanas e desde 1978(10), para ceratocone. No entanto os estudos relativos à técnica para ceratocone oferecem poucos detalhes sobre os resultados.

O presente estudo permitiu maior detalhamento dos resultados pelo fato de se ter adotado tempo total de seguimento de 24 meses para todos os pacientes, com avaliações trimestrais sistemáticas permitindo identificação de modificações corneanas pela retirada das suturas e renovação do epitélio corneal, já que as modificações fisiológicas são observáveis tardiamente ${ }^{(11)}$. Evitou-se o uso de melhor acuidade visual ou acuidade visual final, pela possibilidade de superestimar o número de pacientes com bons resultados e subestimar aqueles com baixa acuidade visual, em relação à possibilidade de modificação desse parâmetro oftalmológico, ao longo do tempo ${ }^{(12)}$.

Os aspectos mais relevantes de nossos resultados foram a redução do astigmatismo com consequente melhora da acuidade visual, representada pelo ganho de linhas de visão, e redução do equivalente esférico, devido ao aplanamento corneano cirúrgico, man- tendo estável a celularidade. No entanto, à medida que houve a acomodação do tecido corneano após a retirada das suturas, ocorreu piora dos parâmetros verificados após seis meses da cirurgia.

Verificou-se redução da acuidade visual, embora não significante, no período de 12 para 24 meses, com piora do equivalente esférico, consoante com a literatura, que demonstra modificações até mesmo imprevisíveis com a retirada das suturas ${ }^{(11)}$. Dentre os fatores a serem considerados para explicar os resultados da pesquisa em foco estão o processo incisional na córnea, as características das suturas e sua relação com o processo cicatricial de uma córnea com ceratocone $^{(13)}$.

Admitindo que a córnea normal tem a conformação de uma hemiesfera, discretamente elíptica ${ }^{(14)}$, é possível comprovar que as forças se distribuem de maneira homogênea tridimensionalmente, devido a um comportamento mecânico elástico aproximadamente linear ${ }^{(15)}$. Esse efeito é determinado pela aparente inextensibilidade do limbo corneal, devido à orientação circunferencial das fibras colágenas ${ }^{(13)}$

No entanto, no ceratocone, as regiões afinadas contêm menor número de lamelas colágenas, que deslizam entre si, e falha mecânica das fibras, o que promove alteração das propriedades mecânicas do tecido. Com isso, o poder dióptrico é alterado. Como a combinação do efeito de afinamento tecidual com a degradação das propriedades sobre o poder dióptrico não é linear, com o passar do tempo as interações entre esses fatores exacerbam a falha local progressiva das fibras colágenas, o que causa aumento das deformações na região corneal afinada, de forma imprevisível|(13). 
Tabela 3. Distribuição das variações dos valores ceratométricos de 18 olhos submetidos a CPAIR, segundo época das aferições Recife - agosto 2004 - junho 2009

\begin{tabular}{|c|c|c|c|c|c|c|}
\hline \multicolumn{2}{|c|}{$\begin{array}{l}\text { Variação dos valores } \\
\text { ceratométricos }\end{array}$} & $\begin{array}{l}\text { de } 6 \text { meses - } \\
\text { inicial }\end{array}$ & $\begin{array}{l}\text { de } 12 \text { meses - } \\
\text { inicial }\end{array}$ & $\begin{array}{l}\text { de } 24 \text { meses - } \\
\text { inicial }\end{array}$ & $\begin{array}{c}\text { de } 12 \text { meses - } \\
6 \text { meses }\end{array}$ & $\begin{array}{l}\text { de } 24 \text { meses - } \\
12 \text { meses }\end{array}$ \\
\hline \multicolumn{7}{|c|}{ Variação do K1 (Dp) } \\
\hline \multirow[t]{2}{*}{ Redução } & $-30,00$ a $-10,00$ & 8 & 6 & 3 & 0 & 0 \\
\hline & $-9,99$ a $-0,01$ & 8 & 9 & 14 & 5 & 5 \\
\hline \multirow[t]{2}{*}{ Aumento } & $+0,01 \mathrm{a}+10,00$ & 0 & 1 & 0 & 10 & 12 \\
\hline & $+10,01 \mathrm{a}+20,00$ & 0 & 0 & 0 & 1 & 0 \\
\hline \multicolumn{7}{|c|}{ Variação do K2 (Dp) } \\
\hline \multirow[t]{2}{*}{ Redução } & $-30,00$ a $-10,00$ & 5 & 6 & 2 & 0 & 0 \\
\hline & $-9,99$ a $-0,01$ & 12 & 11 & 14 & 5 & 5 \\
\hline \multirow[t]{2}{*}{ Aumento } & $+0,01 \mathrm{a}+10,00$ & 1 & 0 & 1 & 13 & 12 \\
\hline & $+10,01 \mathrm{a}+20,00$ & 0 & 1 & 0 & 0 & 0 \\
\hline \multicolumn{7}{|c|}{ Variação do K médio (Dp) } \\
\hline \multirow[t]{2}{*}{ Redução } & $-30,00$ a $-10,00$ & 8 & 6 & 2 & 0 & 0 \\
\hline & $-9,99$ a $-0,01$ & 7 & 11 & 14 & 4 & 4 \\
\hline Aumento & $+0,01 \mathrm{a}+10,00$ & 1 & 1 & 1 & 12 & 13 \\
\hline
\end{tabular}

Nota: Não se pode aferir K1 de dois pacientes, na $2^{\underline{a}}$ avaliação e um paciente na $3^{\underline{a}}$ e $4^{\underline{a}}$ avaliações, $K 2$, de um paciente na $4^{\underline{a}}$ avaliação

Tabela 4. Distribuição das variações da espessura e do número de células corneana de 18 olhos submetidos a CPAIR, segundo época das aferições - Recife - agosto 2004 - junho 2009

\begin{tabular}{|c|c|c|c|c|c|c|}
\hline $\begin{array}{l}\text { Diferenças } \\
\text { corneanas }\end{array}$ & & $\begin{array}{l}\text { de } 6 \text { meses - } \\
\text { inicial }\end{array}$ & $\begin{array}{l}\text { de } 12 \text { meses - } \\
\text { inicial }\end{array}$ & $\begin{array}{c}\text { de } 24 \text { meses - } \\
\text { inicial }\end{array}$ & $\begin{array}{c}\text { de } 12 \text { meses - } \\
6 \text { meses }\end{array}$ & $\begin{array}{c}\text { de } 24 \text { meses - } \\
12 \text { meses }\end{array}$ \\
\hline \multicolumn{7}{|c|}{ Variação da espessura corneana $(\mu)$} \\
\hline \multirow[t]{2}{*}{ Redução } & $-150,00$ a $-100,00$ & 0 & 0 & 1 & 0 & 0 \\
\hline & $-99,99$ a $-1,00$ & 9 & 14 & 6 & 10 & 5 \\
\hline Manutenção & 0 & 0 & 0 & 0 & 1 & 1 \\
\hline \multirow[t]{2}{*}{ Aumento } & $+1,00$ a $+100,00$ & 8 & 3 & 6 & 6 & 8 \\
\hline & $+100,01 \mathrm{a}+160,00$ & 0 & 0 & 2 & 0 & 2 \\
\hline \multicolumn{7}{|c|}{ Variação do número de células corneanas } \\
\hline \multirow[t]{2}{*}{ Redução } & $-1500,00$ a $-200,00$ & 6 & 8 & 12 & 3 & 7 \\
\hline & $-199,00$ a $-1,00$ & 2 & 2 & 0 & 2 & 3 \\
\hline Manutenção & 0 & 2 & 0 & 0 & 1 & 0 \\
\hline \multirow[t]{2}{*}{ Aumento } & $+1,00$ a $+200,00$ & 1 & 2 & 0 & 3 & 3 \\
\hline & $+201,00 \mathrm{a}+8500,00$ & 1 & 2 & 1 & 3 & 4 \\
\hline
\end{tabular}

Nota: Não se pode aferir espessura corneana de um paciente, na $2^{\underline{a}}$ e $3^{\underline{a}}$ avaliações e de três pacientes na $4^{\underline{a}}$; como também o número de células de seis pacientes na $2^{\underline{a}}$ avaliação, quatro casos, na $3^{\underline{a}}$ e cinco casos na $4^{\underline{a}}$, devido à irregularidade corneana

Esse fato pareceu explicar as alternâncias entre o aumento e a redução nos parâmetros ceratométricos, quando foi comparada cada aferição à subsequente, demonstrando que, durante o processo cicatricial, a alteração das propriedades mecânicas ocorre de forma imprevisível, diferindo de uma córnea normal, sem ceratocone ${ }^{(16)}$. O aplanamento corneano cirúrgico possivelmente promove um rearranjo das lamelas colágenas na região do ceratocone, cuja associação com a tensão das suturas, acarreta a melhora dos parâmetros ceratométricos. Dessa forma, a retirada das suturas, reduzindo a força de tração, permite que as lamelas colágenas deslizem livremente e, à medida que a região corneal afinada se reorganiza, determina novos parâmetros ceratométricos. É plausível supor que decorridos 24 meses, esse processo de reorganização se estabilize ou sofra apenas pequenas modificações ${ }^{(17)}$.

Poder-se-ia argumentar que a técnica de sutura empregada, com pontos separados, poderia ter contribuído também para os resultados. No entanto Javadi et al. ${ }^{(18)}$, comprovaram que a sutura contínua, interrompida, dupla ou a combinação da sutura contínua e interrompida são comparáveis quanto ao astigmatismo resultante após 12 meses da cirurgia e dois meses após a retirada completa das suturas

A variabilidade das mensurações paquimétricas provavelmente se deveu ao fato da modificação do local de aferição. A paquime- tria é avaliada na região central da córnea. Após a cirurgia, o ponto de aferição foi modificado pela rotação a $180^{\circ}$ do botão corneano, bem como houve o aplanamento corneano pela retirada do crescente de 0,5 mm. Esses fatores contribuíram para as diferenças das médias da curvatura, mas também podem ter atuado na maior variabilidade do desvio padrão das quatro aferições.

Diferente da crítica de Panda et al. ${ }^{(19)}$, relativa ao maior risco de desenvolvimento de glaucoma secundário derivado do "stress" das estruturas angulares, maior mudança da vascularização devido a alteração limbal, a qual pode induzir defeitos epiteliais ou infecção como resultado da persistência das suturas por um longo período, é importante ressaltar que nenhum dos olhos operados apresentou qualquer dessas alterações

Apesar do astigmatismo verificado nos pacientes após 24 meses da cirurgia, deve-se considerar o ganho de linhas de visão identificado no seguimento de 24 meses, posto que esse astigmatismo que não comprometeu, de maneira significante, a qualidade de vida dos pacientes. Além disso, é importante ressaltar os relatos de satisfação feitos pelos pacientes com os resultados obtidos já que, comparativamente à acuidade visual inicial, apenas dois não apresentaram ganho com a cirurgia e possibilidade de retorno ao trabalho.

Durante os primeiros seis meses após a CPAIR, a avaliação oftalmológica coincidiu com o relato de ganho de qualidade de vida 
Quadro 1. Demonstrativo das alterações significantes nos parâmetros oftalmológicos de 18 olhos submetidos a CPAIR, segundo época das aferições - Recife - agosto 2004 - junho 2009

\begin{tabular}{|c|c|c|c|c|c|}
\hline $\begin{array}{l}\text { Diferenças significantes dos } \\
\text { parâmetros avaliados }\end{array}$ & $\begin{array}{l}6 \text { meses } x \\
\text { inicial }\end{array}$ & $\begin{array}{l}12 \text { meses } x \\
\text { inicial }\end{array}$ & $\begin{array}{l}24 \text { meses } x \\
\text { inicial }\end{array}$ & $\begin{array}{l}12 \text { meses } x \\
6 \text { meses }\end{array}$ & $\begin{array}{c}24 \text { meses } x \\
12 \text { meses }\end{array}$ \\
\hline Acuidade visual (logMAR) & $\uparrow$ & $\uparrow$ & $\boldsymbol{\uparrow}$ & & \\
\hline Equivalente esférico & $\uparrow$ & & & $\downarrow$ & \\
\hline Astigmatismo topográfico & $\downarrow$ & $\downarrow$ & & & \\
\hline Valor ceratométrico máximo simulado & $\downarrow$ & $\downarrow$ & $v$ & $\downarrow$ & \\
\hline $\mathrm{K} 1$ & $\downarrow$ & $\downarrow$ & $\downarrow$ & & $\boldsymbol{\uparrow}$ \\
\hline $\mathrm{K} 2$ & $\downarrow$ & $\downarrow$ & $v$ & $\uparrow$ & \\
\hline$K_{\text {médio }}$ & $\downarrow$ & $\downarrow$ & $v$ & & $\boldsymbol{\sim}$ \\
\hline Espessura corneana & & $\downarrow$ & & $\downarrow$ & $\uparrow$ \\
\hline Número de células endoteliais corneanas & $\downarrow$ & & & & \\
\hline
\end{tabular}

$\boldsymbol{\uparrow}=$ aumento significante; $\boldsymbol{\Downarrow}=$ reduçãosignificante

feito pelos pacientes, cuja intensidade era de tal ordem que três deles solicitaram a realização da CPAIR no olho contralateral. No entanto, o seguimento prolongado para 12 e 24 meses permitiu a verificação de uma preocupante redução da acuidade visual, período em que a discordância entre a avaliação clínica e a do paciente se tornou evidente e a decisão da suspensão de novos procedimentos foi adotada, apesar dos resultados encorajadores obtidos com poucas complicações derivadas do procedimento cirúrgico, diferindo dos relatos de resultados com ceratoplastia homóloga ${ }^{(20)}$.

No Colaborative Longitudinal Evaluation of Keratoconus Study (CLEK), o qual envolveu 1.209 pacientes acompanhados por oito anos após cirurgia para ceratocone realizada por várias técnicas, a avaliação de qualidade de vida desses pacientes demonstrou que seu parâmetro para classificar a modificação da visão foi a lembrança da acuidade visual antes do procedimento cirúrgico, do que derivou uma diferença entre a avaliação oftalmológica e a do paciente. Os autores concluíram que as avaliações clínicas tradicionais, tais como a acuidade visual, não traduzem adequadamente o impacto antes e após a cirurgia na qualidade de vida do paciente, podendo gerar aflição ao oftalmologista, em face de uma acuidade visual diferente daquela que seria o ideal, e a satisfação do paciente com o resultado obtido ${ }^{(21,22)}$

A presente pesquisa apresentou limitações que foram ressaltadas por Panda et al. ${ }^{(19)}$ A ceratoplastia eletiva deve oferecer tanto resultados ópticos quanto cosméticos e, na CPAIR, os resultados cosméticos não foram avaliados, porém a distorção palpebral secundária à protrusão corneal é minimizada já que a técnica promove aplanação.

\section{CONCLUSÃO}

A ceratoplastia penetrante autóloga ipsilateral rotacional mostrou-se uma técnica segura, com possibilidade de melhorar a acuidade visual dos pacientes com ceratocone, pela redução do astigmatismo, sem perda significante do número de células corneanas. No entanto não se puderam explicar resultados tão díspares entre os olhos operados, em relação aos parâmetros aferidos.

Parece plausível supor que novos estudos poderão permitir a identificação das características dos ceratocones, responsáveis por perda da acuidade visual ao longo do tempo, diferenciando pacientes para os quais essa técnica cirúrgica poderá ser uma opção terapêutica válida.

\section{REFERÊNCIAS}

1. Endriss D, Cunha F, Ribeiro MP, Toscano J. Ceratoplastias penetrantes realizadas na Fundação Altino Ventura: revisão dos resultados e complicações. Arq Bras Oftalmol. 2003; 66(3):273-7.
2. Langenbucher A, Gusek-Schneider GC, Kus MM, Seitz B. [Topography-based calculation of Keratoconus dimensions]. Klin Monatsbi Augenheilkd. 1999;214(6):372-7. German.

3. Colin J, Velou S. Current surgical options for keratoconus. J Cataract Refract Surg. 2003; 29(2):379-86.

4. Tanure MA, Trindade FC. Ceratoplastia penetrante autóloga ipsilateral com rotação do disco corneano em casos de ceratocone: resultados iniciais. Rev Bras Oftalmol. 2001;60(10): 719-29.

5. Pernambuco. Secretaria Estadual de Saúde. Central de transplantes. Lista única de Pernambuco. Pacientes ativos e inativos a espera de órgãos e tecidos no Estado de Pernambuco [Internet]. Recife: Secretaria Estadual de Saúde; 2011. [citado 2011 abril 30]. Disponível em: http://www.transplantes.pe.gov.br/estatistica_lista.htm

6. Jonas JB, Rank RM, Budde WM. Autologous ipsilateral rotating penetrating keratoplasty. Am J Ophthalmol. 2001;131 (4):427-30. Comment in: Am J Ophthalmol. 2002;133(4):590; author reply $590-1$

7. Melo Filho A, Palmeira FC, Machado MA. Transplante autólogo de córnea: relato de 3 casos. An Paul Med Cir. 2000;127(4):280-3.

8. Verma N, Melengas S, Garap JA. Ipsilateral rotation autokeratoplasty for management of corneal opacities. Aust N Z J Ophthalmol. 1999;27(1):21-5.

9. Montenegro WT. Ceratoplastia penetrante autóloga ipsilateral com ressecção em crescente e rotação do disco corneal em ceratocone anterior [tese]. Belo Horizonte: Universidade Federal de Minas Gerais; 2003.

10. Bourne WM, Brubaker RF. A method for ipsilateral rotational autokeratoplasty. Ophthalmology. 1978;85(12):1312-6.

11. Langenbucher A, Naumann GOH, Seitz B. Spontaneous long-term changes of corneal power and astigmatism after suture removal after penetrating keratoplasty using a regression model. Am J Ophthalmol. 2005:140(1):29-34.

12. DiLoreto DA Jr, Bressler NM, Bressler SB, Schachat AP. Use of best and final visual acuity outcomes in ophthalmological research. Arch Ophthalmol. 2003;121(11):1586-90.

13. Gefen A, Shalom R, Elad D, Mandel Y. Biomechanical analysis of the keratoconic cornea. J Mech Behavior Biomed Mater. 2009:2|(3):224-36.

14. Calossi A. Corneal asphericity and spherical aberration. J Refract Surg. 2007;23(5):505-14. Comment in: J Refract SUrg. 2008;24(2):119-21; author reply 121

15. Pierscionek BK, Asejcyk-Widlicka M, Schachar RA. The effect o changing intraocular pressure on the corneal and scleral curvatures in the fresh porcine eye. $\mathrm{Br} J \mathrm{Ophthalmol}$. 2007;91(6):801-3.

16. Joseph R, Srivastava OP, Pfister RR. Differential epithelial and stromal protein profiles in keratoconus and normal human corneas. Exp Eye Res. 2011;92(4):282-98.

17. Akhtar S, Bron AJ, Salvi SM, Hawksworth NR, Tuft SJ, Meek KM. Ultrastructural analysis of collagen fibrils and proteoglycans in keratoconus. Acta Ophthalmol. 2008;86(7):764-72.

18. Javadi MA, Naderl M, Zare M, Jenaban A, Rabei HM, Anissian A. Comparison of the effect of three suturing techniques on postkeratoplasty astigmatism in keratoconus. Cornea. 2006;25(9):1029-33

19. Panda A, Sudan R, Nainiwal S. Autologous ipsilateral rotating penetrating keratoplasty. Am J Ophthalmol. 2002;133(4):590.; author reply 590-1. Comment in: Am J Ophthalmol. 2001;131(4):427-30

20. Bahar I, Kaiserman I, Srinivasan S, Ya-Ping J, Slomovic AR, Rootman DS. Comparison of three different techniques of corneal transplantation for keratoconus. Am J Ophthalmol. 2008; 146(6):905-12.

21. Kymes SM, Walline JJ, Zadnik K, Sterling J, Gordon MO; Collaborative Longitudinal Evaluation of Keratoconus Study Group. Changes in the quality-of life of people with keratoconus. Am J Ophthalmol. 2008;145(4):611-7.

22. Kymes SM, Walline JJ, Zadnik K, Gordon MO; Collaborative Longitudinal Evaluation of Keratoconus Study Group. Quality of life in keratoconus. Am J Ophthalmol. 2004;138(4): 527-35. Comment in: Am J Ophthalmol. 2004;138(4):63-8. 